\title{
Chronic Unpredictable Mild Stress Accelerates the Growth of Bladder Cancer in a Xenograft Mouse Model
}

This article was published in the following Dove Press journal: Psychology Research and Behavior Management

\author{
Qidong Zhou ${ }^{\mathrm{I}, 2}$ \\ Weihong Ding ${ }^{1,2}$ \\ Zhiyu Qian ${ }^{1,2}$ \\ Guangliang Jiang ${ }^{3}$ \\ Chuanyu Sun ${ }^{1,2}$ \\ $\mathrm{Ke} X \mathrm{u}^{1,2}$
}

'Department of Urology, Huashan Hospital, Fudan University, Shanghai, People's Republic of China; ${ }^{2}$ Fudan Institute of Urology, Huashan Hospital, Fudan University, Shanghai, People's Republic of China; ${ }^{3}$ Department of Urology, Ruijin Hospital, Medical School of Shanghai Jiao Tong University, Shanghai, People's Republic of China
Correspondence: $\mathrm{Ke} \mathrm{Xu}$ Department of Urology, Huashan Hospital, Fudan University, No. 12 Middle Urumqi Road, Shanghai 200040, People's Republic of China

$\mathrm{Tel} / \mathrm{Fax}+862152887080$

Email drkexu@।63.com
Objective: Chronic psychological stress is common in patients with bladder cancer. An increasing number of evidence demonstrated that psychiatric disorder leads to worse prognostic outcomes in bladder cancer. This study was to investigate the effects of chronic psychological stress on the growth of bladder cancer and its potential mechanisms.

Methods: A xenograft mouse model was established by subcutaneously implanting the human bladder cancer cell line T24 into nude mice. All of the tumor-bearing mice $(\mathrm{N}=20)$ were randomly separated into two groups. Mice in the control group were subjected to normal feeding conditions, while in another group, a chronic unpredictable mild stress (CUMS) model was established, in which mice were exposed to various types of stressors. Various analyses were performed on parameters including the tumor volume, tumor weight, expression of Caspase-3 and VEGF, proportion of Ki-67 positive cells (Ki-67 index), microvessel density (MVD) and serum concentrations of epinephrine and cortisol.

Results: In the CUMS group, the growth of transplanted tumors was distinctly accelerated, with the weight of removed tumors at the end of experiment increased by $34.07 \%$ compared to that of the control. Serum levels of epinephrine and cortisol determined by ELISA were significantly increased by CUMS. Immunohistochemistry and Western blot analysis showed that the expression of Caspase-3 was downregulated, whereas the expression of VEGF was upregulated in the CUMS group. Meanwhile, CUMS could increase the Ki-67 index and MVD.

Conclusion: Our research supports the hypothesis that CUMS could affect the growth of bladder cancer in nude mice, indicating that the intervention of chronic psychological stress may be a possible therapeutic strategy for bladder cancer.

Keywords: bladder cancer, chronic unpredictable mild stress, tumor growth, cell proliferation, angiogenesis

\section{Introduction}

Bladder cancer ranks ninth most common among types of cancer worldwide. Approximately 430,000 new bladder cancer cases and 165,000 bladder cancer deaths occur worldwide per year. ${ }^{1}$ As a physically and psychologically debilitating disease, bladder cancer usually leads to severe emotional distress such as anxiety, depression and suicidality. ${ }^{2}$ The diagnosis of cancer, repeated treatment, worsening of symptoms, changing state of illness, and negative life events could easily cause chronic psychological stress in bladder cancer patients. Essentially, it is reported that the incidence of anxiety and depression in patients with bladder cancer is significantly higher than that of other tumor patients in China. ${ }^{3}$ 
Some studies have indicated that depressive symptoms and major depressive disorder are associated with increased mortality in bladder cancer patients. ${ }^{4}$ Additionally, substantial evidence demonstrated that the diagnosis of posttreatment psychiatric disorder resulted in significantly worse overall survival and cancer-specific survival in bladder cancer. ${ }^{5}$ Chronic psychological stress has been shown to intervene in immune function and play a role in the tumor growth and progression in diverse carcinomas. ${ }^{6-8}$ However, the effects of chronic psychological stress on the biological behaviors of bladder cancer and possible underlying mechanisms are unknown.

It has been revealed that the complex mechanisms of the influence of chronic stress on cancer cells are related to the sympathetic nervous system (SNS) and the hypothalamicpituitary-adrenal (HPA) axis, which further act on the immune system and microenvironment of tumors. The animal models of chronic stress have become indispensable in order to discuss the relationship between chronic psychological stress and cancer growth under better-controlled conditions. In those previous studies, chronic stress models using one single stressor, such as the chronic restraint model, have been widely adopted. ${ }^{6,7,9}$ Unfortunately, the long-term repetitive stressor can make animals adaptive. Therefore, a model capable of better simulating various unpredictable stressors and chronic psychological stress in bladder cancer patients is required. ${ }^{10}$

In the current study, we introduced the chronic unpredictable mild stress model (CUMS) in which different types of stressors were applied and the xenograft model of bladder cancer in nude mice to investigate the effects of chronic stress on bladder cancer growth. We examined the serum levels of epinephrine and cortisol in mice exposed to CUMS. Meanwhile, the expressions of Caspase-3 and VEGF, Ki-67 index and microvessel density (MVD) in the transplanted tumors were analyzed to preliminarily explore the possible mechanisms.

\section{Materials and Methods}

\section{Cell Lines and Animals}

The human bladder cell line T24 was obtained from the cell bank of the Chinese Academy of Sciences (Shanghai, China). Cells were cultured in McCoy's 5a medium (Invitrogen) supplemented with 10\% fetal bovine serum (FBS; Thermo Fisher Scientific, Waltham, MA, USA) and penicillin-streptomycin at $37^{\circ} \mathrm{C}$ in an atmosphere of $5 \%$ $\mathrm{CO}_{2}$.
Male BALB/c-nu mice weighting 15-18 g (3-4 weeks old) were purchased from the Shanghai SLAC Laboratory Animal Co., Ltd. (Shanghai, China). All animal experiments were approved by the animal ethics committee of Fudan University (Ethical approval number: 2019-HSYY-JS-240) and conformed to the guidelines of the National Research Council for Laboratory Animal Care in Research.

\section{In vivo Implantation of T24 Cells}

Mice were group housed under SPF conditions and were allowed to adapt to the laboratory environment for 1 week prior to start of the experiments. T24 cells harvested after brief incubation in $0.25 \%$ trypsin and $0.02 \%$ EDTA were injected subcutaneously into the right lateral chest wall of nude mice at a concentration of $3 \times 10^{6}$ cells $/ 0.2 \mathrm{~mL}$ of PBS. The needle was kept subcutaneously after injection for $30 \mathrm{~s}$ to prevent the liquid from flowing back.

\section{Chronic Unpredictable Mild Stress Model}

Mice were randomly divided into CUMS ( $\mathrm{n}=10)$ or control $(n=10)$ groups after the in vivo implantation of tumor cells. The CUMS procedure was conducted as previously described $^{11-13}$ but adjusted slightly. Briefly, stress regimes were administered, consisting of the following eight stressors: food deprivation (24 h), water deprivation (24 h), tail pinch ( $5 \mathrm{~min}, 1 \mathrm{~cm}$ from the distal portion of the tail), cold swimming $\left(3 \mathrm{~min}\right.$ at $\left.4^{\circ} \mathrm{C}\right)$, physical restraint $(2 \mathrm{~h})$, moist bedding $(6 \mathrm{~h}), 30^{\circ}$ cage tilt $(12 \mathrm{~h})$ and overnight stroboscopic lighting. Two stressors were administered daily in a random order for 28 days consecutive, with the same stressor not applied on two consecutive days. Mice in the control group were not subjected to any of these stressors throughout the experiment. The long diameter (a) and short diameter (b) of tumors were measured using calipers every 4 days in each mouse, and volumes were approximated by the formula $\mathrm{V}=\mathrm{a} \times \mathrm{b}^{2} / 2{ }^{6}$ All of the mice were weighed and sacrificed by decapitation 28 days after tumor implantation. The removed tumors were weighed, and the blood samples were collected for further analysis.

\section{Measurement of Epinephrine and Cortisol}

The blood samples of mice were all collected immediately after sacrifice at approximately 9:00 a.m., as stress hormones can vary with circadian rhythms. The serum levels of epinephrine and cortisol were determined by enzyme-linked immunosorbent assay (ELISA). Plasma was separated by centrifugation and stored at $-80^{\circ} \mathrm{C}$ for later analysis of the epinephrine and cortisol concentrations. Samples were tested 
using epinephrine (Alpco Diagnostics, Salem, NH, USA) and cortisol (R\&D Systems, Minneapolis, MN, USA) ELISA kits according to the manufacturers' instructions. The absorbance was measured at $450 \mathrm{~nm}$ using a microplate reader.

\section{Histology and Immunohistochemical Assay}

Bladder tumor xenografts were removed, fixed in $10 \%$ formalin and embedded in paraffin. Sections were stained with hematoxylin and eosin (HE) to evaluate the histological changes using a light microscope (H550S, Nikon, Japan). In addition, immunohistochemical analysis of these sections was performed using specific antibodies for Ki-67, Caspase-3 and VEGF (Proteintech, 1:100). Briefly, sections were incubated in 3\% hydrogen peroxide for $15 \mathrm{~min}$ and blocked for $30 \mathrm{~min}$ at room temperature using horse blocking buffer. Sections were then incubated with primary antibodies overnight at $4{ }^{\circ} \mathrm{C}$. The goat antirabbit HRP conjugated secondary antibodies were used to detect primary antibodies. Color was developed with fresh $\mathrm{DAB}$ solution for $5 \mathrm{~min}$. Five random fields at 200x magnification were examined for each tumor, and the mean optical density was measured using Image-Pro plus 6.0 software. The quantification of cell proliferation was based on Ki-67 staining, and the results were expressed as ratios of positive cells/total cells (Ki-67 index). Five sections per five random tumor tissues were quantified.

\section{Microvessel Density Assessment}

To quantify the microvessel density (MVD) in xenografts, the tumor sections were immunostained with mouse antimonoclonal CD34 antibody (Proteintech, 1:100) as the primary antibody. Counterstaining was performed with hematoxylin. Any brown-stained endothelial cell or endothelial cell cluster, clearly separate from adjacent tissue elements, was considered to be a single, countable microvessel under microscopy. Five sections per five random tumor tissues were quantified. The sections were scanned at low power (40x magnification) to identify five areas rich in microvessels. Then, in each area, individual microvessels were counted at high power (200x magnification) to obtain a vessel count. The average vessel count in the five randomized areas was taken as the MVD. Data extraction was conducted in a blinded fashion so that the researchers who performed analysis did not know which group the tumor sections belonged to.

\section{Protein Extraction and Western Blotting}

Xenograft proteins were extracted with RIPA buffer and quantified using the BCA method. Then, protein samples were separated by $10 \%$ SDS-PAGE electrophoresis and transferred to PVDF membranes (Millipore). The membrane was blocked in $5 \%$ nonfat milk with $0.1 \%$ Tween20. The membrane blots were incubated with primary Caspase- 3 and VEGF antibodies (1:1000, Cell Signaling Technology) overnight at $4^{\circ} \mathrm{C}$ and with goat anti-rabbit HRP-conjugated secondary antibodies for $1 \mathrm{~h}$. Then, the membranes were incubated in ECL solution, and immunodetection bands were photographed and scanned. Data were from pooled tumors of five random xenografts in each group, and three technical replicates were performed.

\section{Statistical Analysis}

SPSS software (version 21.0, SPSS Inc., Chicago, IL, USA) was used to analyze the experimental data. The data were presented as the mean $\pm \mathrm{SD}$ and analyzed by the Student's two-tailed $t$-test between two groups. The individual time points of tumor growth were also compared by the Student's $t$-test. A P-value less than 0.05 was considered statistically significant.

\section{Results}

\section{Chronic Unpredictable Mild Stress Promoted Bladder Cancer Growth in Nude Mice}

Mice in the CUMS group showed irritability and anxiety for the first three days. Then, the mice were generally inactive with reduced movement in the following days until the end of the experiment. Approximately 4-5 days after the inoculation of T24 cells, a tumor mass began to appear in the right lateral chest wall in both groups. However, the tumor growth in the CUMS group presented a more rapid tendency than that in the control group. Significantly, the tumor volumes measured on the 24th and 28th day in CUMS group were larger than those in the control group (Figure 1A). There was no difference in the body weight of mice between the two groups after the experiment, while the weights of removed T24 xenografts were $1.35 \pm 0.35 \mathrm{~g}$ and $1.81 \pm 0.52 \mathrm{~g}$ in the two groups, respectively (Figure 1B, Table 1). We calculated that the tumor growth rate was $34.07 \%$, indicating that CUMS obviously promoted the growth of bladder tumor xenografts. 
A

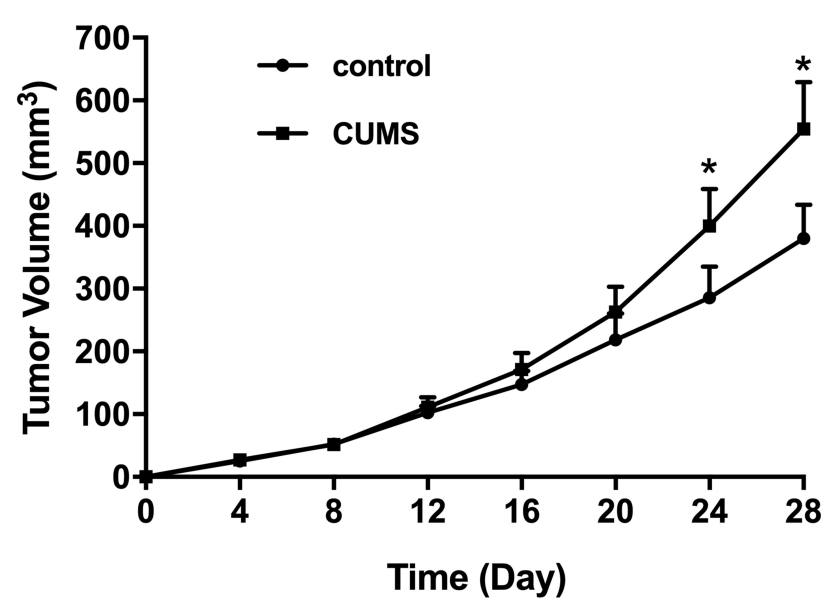

B

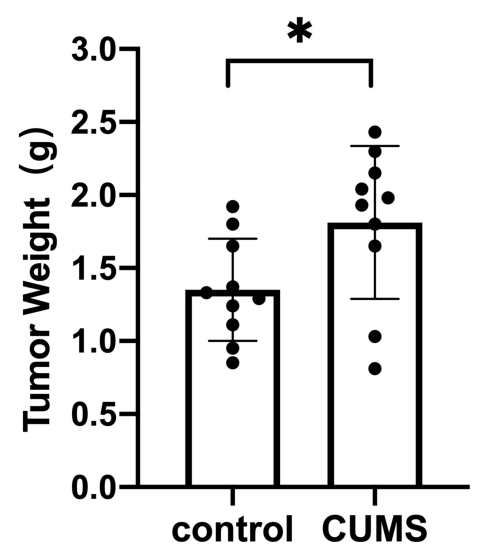

Figure I Tumor volumes and weight measured in CUMS group and control. T24 bladder cells were inoculated into the nude mice and were allowed to grow with or without CUMS for 28 days. (A) Tumor volumes measured at a 4 day interval. (B) excised tumor weight among two groups for each biological replicate $(n=10)$. $*_{p}<0.05$, CUMS group vs control.

\section{Chronic Unpredictable Mild Stress} Increased the Serum Levels of Epinephrine and Cortisol

To verify the activation of the HPA axis and SNS, the serum levels of epinephrine and cortisol in nude mice were measured by ELISA assays. The concentration of epinephrine was $174.85 \pm 25.04 \mathrm{pg} / \mathrm{mL}$ in the control group

Table I Weight of Mice and Removed Tumors After Experiments in Both Groups

\begin{tabular}{|c|c|c|}
\hline Data & $\begin{array}{l}\text { Control Group (g, } \\
\text { Mean } \pm S D, n=10)\end{array}$ & $\begin{array}{l}\text { CUMS Group (g, } \\
\text { Mean } \pm S D, n=10)\end{array}$ \\
\hline Weight of mice & $21.74 \pm 1.26$ & $22.30 \pm 0.79$ \\
\hline Weight of tumors & $1.35 \pm 0.35$ & $\mathrm{I} .8 \mathrm{I} \pm 0.52$ \\
\hline Tumor growth rate & & $34.07 \%$ \\
\hline
\end{tabular}

and $234.58 \pm 36.00 \mathrm{pg} / \mathrm{mL}$ in the CUMS group. There was a significant difference between the two groups (Figure 2). Furthermore, the serum cortisol level in the CUMS group $(86.86 \pm 20.16 \mathrm{ng} / \mathrm{mL})$ was significantly higher than that in the control group $(49.45 \pm 12.29 \mathrm{ng} / \mathrm{mL})$. Thus, the multiple stressors applied to the nude mice caused general HPA and SNS stimulus characteristics of chronic stress.

\section{Chronic Unpredictable Mild Stress Changed the Levels of VEGF and Caspase-3 Expression in Xenografts}

Bladder tumor xenografts were histologically confirmed by HE staining in both groups (Figure 3A). VEGF is a signaling protein generated by cells, which can stimulate angiogenesis and thereby promote tumor growth. ${ }^{14} \mathrm{We}$ analyzed the VEGF protein levels in xenografts using IHC and Western blot. The stained images showed that, compared with the control group, the VEGF protein expression in the CUMS group was significantly increased (Figure 3B and D). Additionally, the average band intensity of VEGF shown in Figure 3F was increased in specimens of stressed mice $(p=0.042)$, indicating that CUMS could upregulate the expression of VEGF.

Tumor growth is considered to be the result of abnormal cell proliferation and normal apoptosis dysfunction. The inhibition of apoptosis function is closely related to the change of caspase-3 protein expression. A significant decrease in caspase-3 levels was detected by IHC in the CUMS group (Figure 3C and E). The reduced expression of caspase-3 was also confirmed by Western blot analysis (Figure 3F), showing significant differences between the two groups.

\section{Chronic Unpredictable Mild Stress Increased the Proportion of Ki-67 Positive Cells and MVD in Xenografts}

$\mathrm{Ki}-67$ is an established marker of cell proliferation, which is present during the cell cycle. We examined the cell proliferation rates in xenografts of each groups by Ki-67 staining. Only the nuclear staining result was used to interpret positivity for Ki-67, and the results were expressed as ratios of positive cells/total cells. In our experiment, cell proliferation rates were significantly higher in the CUMS group than that in control mice (Figure 4A and B). These data suggested the increased susceptibility of bladder cancer to CUMS-induced proliferation.

A similar effect was observed with respect to the angiogenesis of tumors. CD34 is specifically expressed 

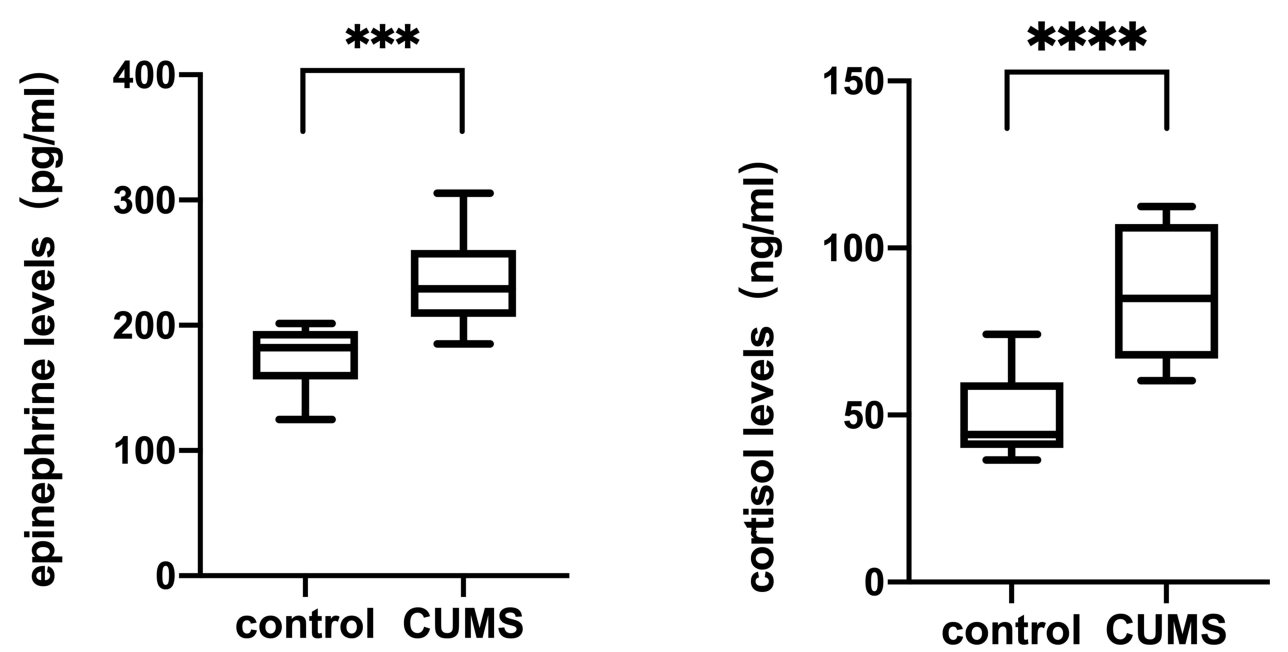

Figure 2 Epinephrine and cortisol levels determined in CUMS group and control. Blood samples were obtained at the end of the experiment and serum concentrations of epinephrine and cortisol were detected by ELISA. ${ }^{* * *} \mathrm{p}<0.001$, ${ }^{* * * *} \mathrm{p}<0.0001$, CUMS group vs control.

A

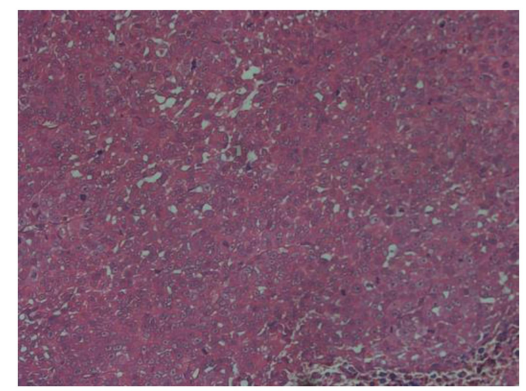

B

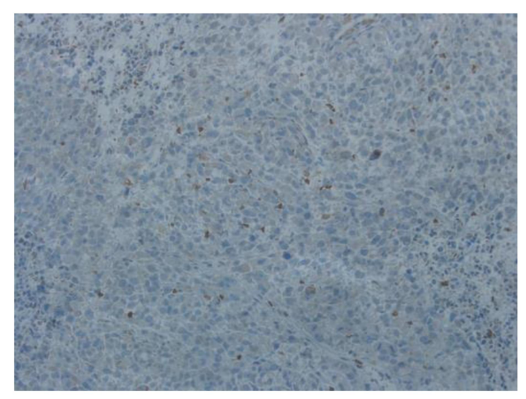

C

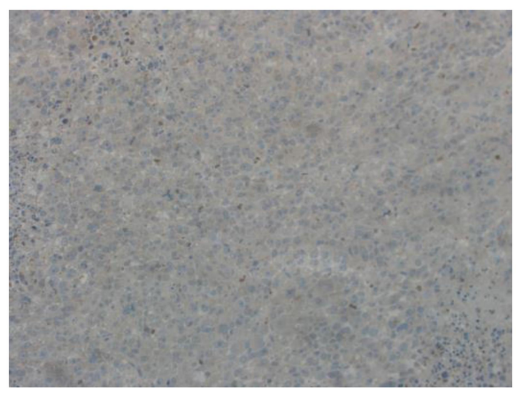

CUMS

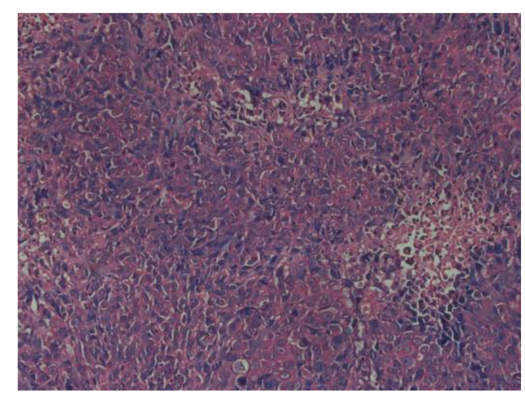

CUMS

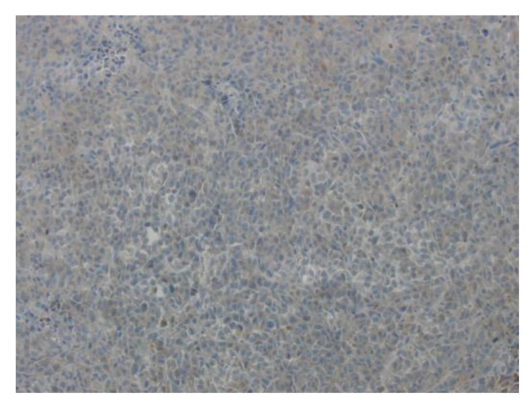

CUMS

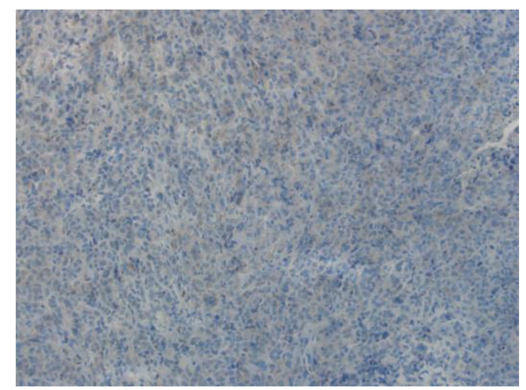

D
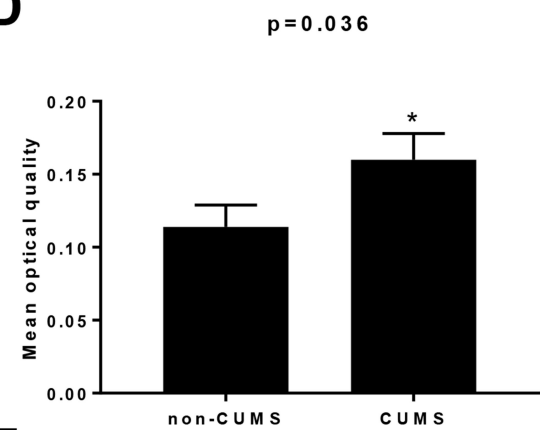

E

$p=0.016$

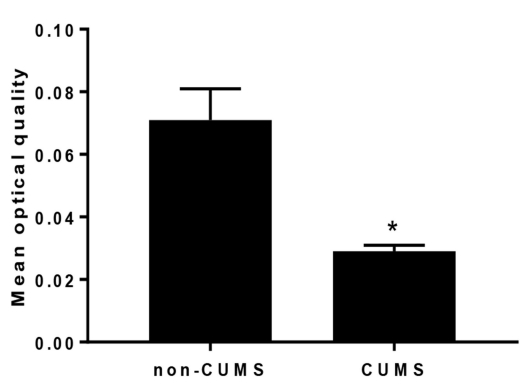

$\mathbf{F}$

Caspase-3

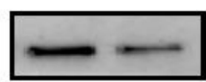

VEGF

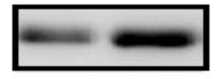

$\beta$-actin

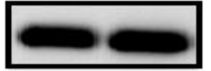

control CUMS

Figure 3 CUMS changed the expression of VEGF and Caspase-3 expression in xenografts. (A) HE staining of tumor tissue of two groups $(\times 200)$. (B) Immunohistochemical staining targeting VEGF $(\times 200)$. (C) Caspase-3 immunohistochemical staining $(\times 200)$. (D) Mean optical quantity of VEGF staining. (E) Mean optical quantity of Caspase-3 staining. (F) Western blot analyses of VEGF and Caspase-3 expression in T24 xenografts. ${ }^{*} p<0.05$, CUMS group vs control. 
A

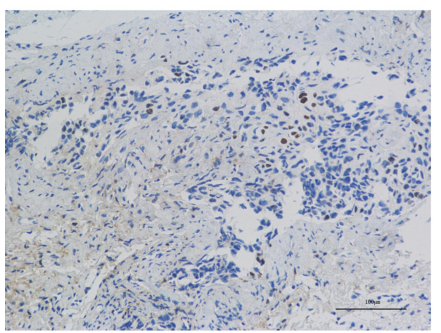

C

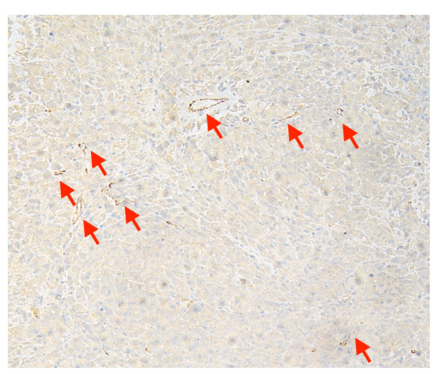

CUMS

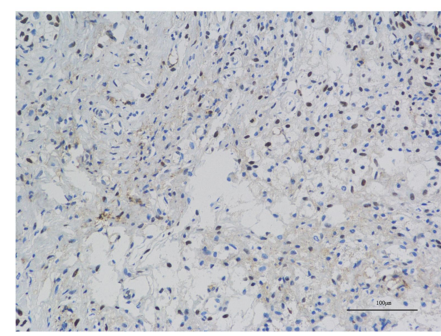

CUMS

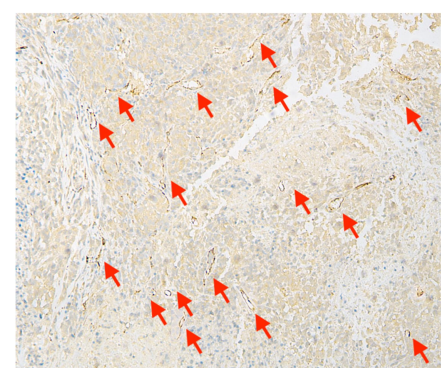

B

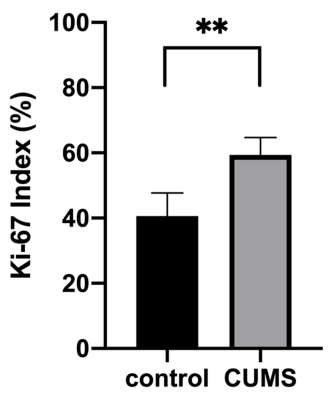

D

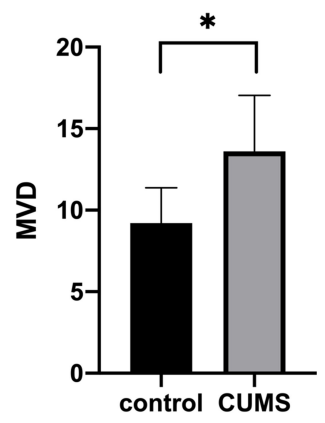

Figure 4 CUMS increased the Ki-67 index and MVD in xenografts. (A) Immunohistochemical staining targeting Ki-67 ( $\times 200)$. (B) The percentage of cells positive for Ki-67 staining was presented as a Ki-67 index. (C) Immunohistochemical staining targeting CD34 $(\times 200)$. Microvessels were indicated with red arrows. (D) The average vessel count in the five areas $(\times 200)$ was taken as the MVD. ${ }^{*} \mathrm{p}<0.05,{ }^{*} \mathrm{p}<0.01$, CUMS group vs control.

on vascular endothelial cells of tumor tissues, which is related to the proliferation and migration of endothelial cells. Therefore, the MVD count using CD34 staining is considered to be an indicator of tumor neo-angiogenesis. As shown in Figure 4C and D, the MVD of bladder cancer xenografts obtained from CUMS mice was obviously higher when compared to that of the control group. The difference was statistically significant, showing that CUMS may exert an important role in tumor angiogenesis and development.

\section{Discussion}

In this research, we mainly investigated whether chronic psychological stress could affect the development of bladder cancer xenografts in nude mice by introducing the CUMS model. Up to $85 \%$ of bladder cancer patients present with non-muscle invasive bladder cancer (NMIBC). ${ }^{15}$ Throughout the entire disease process of NMIBC, patients often experience psychological stress brought on by tumor diagnosis, repetitive treatment, tumor recurrence and progression, and other negative events, which may lead to adverse emotions such as depression, anxiety and fear. The CUMS model is a widely validated and applied model of depression, which leads to anhedonia in animals. ${ }^{10}$ Longterm repetition of the same kind of stress stimulation may make the animals adaptive. However, CUMS involves a series of unpredictable mild stressors to which animals are exposed for a period of weeks to months (at least 2 weeks). In our study, we selected eight different types of stressors, which made the mice not only unable to predict the occurrence of stimulation but also prevented adaptation, thus ensuring the unpredictability and diversity of the chronic stress state. In addition, these mild stressors with low-intensity in the CUMS model could better simulate various life pressures encountered by patients with bladder cancer than those chronic stress models with only one stressor.

Cancers can threaten both human physical and mental health, and many studies have paid more attention to the role of social psychological factors in the development and prognosis of cancers. ${ }^{16,17}$ We explored whether CUMS could have an impact on the growth of subcutaneous xenografts of bladder cancer in nude mice in the current study. The results showed that the growth of transplanted tumors in the CUMS group was faster than that in control group. The tumor volumes measured on the mice of two groups were significantly different, and the average tumor weight of mice exposed to stress increased by $34.1 \%$ compared to the control. The differences in tumor growth were not observed early in tumor initiation but instead 
later. We surmised that this may be due to a cumulative effect of CUMS. Initially, a single or small amount of stimulation had no apparent effect on tumor growth. With the prolongation of time and the increase of the stress times, there were obvious differences in tumor growth. Clinically, the adverse effects of chronic psychological factors on bladder cancer patients may also be persistent and accumulated. Moreover, we found that the serum levels of epinephrine and cortisol were evidently increased in stressed mice.

The HPA axis and SNS play a vital role in regulating tumor growth, but the effects of stress-related hormones on animal models of bladder cancer are poorly understood. Recent studies have found that the levels of epinephrine and norepinephrine generated by SNS have increased continuously in preclinical models of chronic stress in ovarian and peritoneal tissues. ${ }^{18,19}$ The increase of these hormone levels is related to the increase of tumor loads, which is mediated by the tumor enhancement of angiogenesis. Furthermore, the concentration of catecholamine in the circulating plasma of cancer patients provides crucial information related to tumor prognosis. ${ }^{7,19}$ Catecholamines also affect the various steps necessary for tumor metastasis, including invasion and migration. In vitro, NE promoted the migration of colon cancer cells, which could be inhibited by $\beta$-adrenergic receptor ( $\beta$-AR) blockers. ${ }^{20}$ Another major neuroendocrine response to stress is through the activation of the HPA axis, which is indispensable in the process of stress adaptation. ${ }^{19}$ The results showed that the level of endogenous glucocorticoid increased in the stress environment, and glucocorticoid was involved in thymus degeneration induced by stress. The effects of NE and cortisol on the proliferation of oral squamous cell carcinoma have been studied. ${ }^{6,21}$ Specifically, NE and isoproterenol can significantly promote the proliferation of oral squamous cell carcinoma, while the effect of cortisol varies with the concentration of the hormone. It is also found that the effect of cortisol on VEGF in ovarian cancer cells is different, which depends on the hormone level. ${ }^{22}$ More importantly, evidence suggested that the role of glucocorticoid on the growth of bladder cancer may be related to the hormone levels. Experiments in vitro and in vivo discovered that glucocorticoid enhanced the receptor activity mediated by the glucocorticoid receptor (GR), promoted the proliferation of bladder tumor cells and inhibited their apoptosis, but the GR pathway has dual effects on inhibiting the progression of bladder cancer. ${ }^{23}$

Then, we further explored the possible mechanism of CUMS promoting the growth of bladder cancer xenografts in nude mice. The expressions of Caspase- 3 and VEGF in xenografts were detected. In the CUMS group, the expression of Caspase-3 was decreased, whereas the expression of VEGF was increased. Meanwhile, CUMS could increase the proportion of Ki-67 positive cells and MVD. Previous research has detected the expression of Caspase3 in bladder transitional cell carcinoma and normal bladder mucosa. The results showed that compared with normal bladder mucosa, the positive rate of caspase-3 protein expression in bladder transitional cell carcinoma was significantly decreased, and the degree of downregulation increased with the deepening of tumor malignancy, suggesting that caspase-3 protein may be related to the characteristics of tumor apoptosis resistance and the degree of malignancy. ${ }^{24}$ Caspase- 3 is closely related to bladder cancer and interacts with various genes. It is found that the expressions of Bcl-2, p53, survivin and caspase-3 were associated with a high recurrence rate of bladder cancer and disease-specific mortality after radical cystectomy. ${ }^{25}$ On the other hand, several studies have confirmed that $\mathrm{Ki}$ 67 is strongly associated with recurrence and overall survival and identified it as an independent prognostic indicator for bladder cancer. ${ }^{26-28}$ Therefore, we speculate that the active proliferation and limited apoptosis of bladder cancer cells play an important role in the growth of xenografts promoted by CUMS.

In the absence of adequate blood supply, solid tumors cannot continue to grow in an unlimited range. Increased VEGF expression could accelerate tumor growth and metastasis. ${ }^{29}$ In the orthotopic model of ovarian cancer, chronic restraint stress increased the tumor load, while the secretion of VEGF in the tumor increased, indicating that chronic stress may promote tumor development by increasing the blood supply. ${ }^{30}$ In addition to stimulating the migration, proliferation and proteolytic activity of endothelial cells, VEGF is capable of interfering with the functional maturation of dendritic cells and T cells, suggesting that this pro-angiogenic factor may have an effect on the tumor immune response. A clinical study showed that there was a correlation between a higher social support level and a lower serum VEGF level in patients with ovarian cancer [38]. In addition, a high level of social support is associated with a low level of interleukin-6 (IL-6) in peripheral blood and ascites of patients with ovarian cancer, which is also a crucial factor for angiogenesis. ${ }^{31}$ Catecholamines have been shown to increase in adipose tissue and various cancer cell lines, causing the upregulation VEGF or ADRB2 via the $\beta$-AR-cAMP-PKA pathway. ${ }^{22,32}$ This effect can be 
terminated by propranolol, a $\beta$-AR blocker, but induced by isoproterenol. However, there are few studies on the role of $\beta$-AR in bladder cancer. Therefore, further research is needed to provide a more detailed understanding of the mechanism(s). Moreover, additional tumor cell lines, separate data collection for male and female mice and the impact this may have on the outcome are required to refine this study.

Accordingly, our study revealed that CUMS had adverse impact on the growth of subcutaneous bladder cancer xenografts in nude mice. The potential mechanisms were related to the promotion of cell proliferation and angiogenesis in the tumor microenvironment affected by increasing catecholamine and cortisol levels. These results suggested that the intervention of chronic psychological stress may be a possible and promising therapeutic strategy for bladder cancer.

\section{Acknowledgments}

This work was supported by the National Natural Science Foundation of China (No.81372756), Science and Technology Commission of Shanghai Municipality (No. 19ZR1408000).

\section{Disclosure}

The authors report no conflicts of interest for this work.

\section{References}

1. Fernandez MI, Brausi M, Clark PE, et al. Epidemiology, prevention, screening, diagnosis, and evaluation: update of the ICUD-SIU joint consultation on bladder cancer[J]. World J Urol. 2019;37(1):3-13.

2. Vartolomei L, Vartolomei MD, Shariat SF. Bladder Cancer: depression, Anxiety, and Suicidality Among the Highest-risk Oncology Patients[J]. Eur Urol Focus. 2019.

3. Li M, Wang L. The Associations of Psychological Stress with Depressive and Anxiety Symptoms among Chinese Bladder and Renal Cancer Patients: the Mediating Role of Resilience[J]. PLoS One. 2016;11(4):e154729.

4. Lin J, Blalock JA, Chen M, et al. Depressive symptoms and short telomere length are associated with increased mortality in bladder cancer patients[J]. Cancer Epidemiol Biomarkers Prev. 2015;24 (2):336-343.

5. Jazzar U, Yong S, Klaassen Z, et al. Impact of psychiatric illness on decreased survival in elderly patients with bladder cancer in the United States[J]. Cancer. 2018;124(15):3127-3135.

6. Xie H, Li C, He Y, et al. Chronic stress promotes oral cancer growth and angiogenesis with increased circulating catecholamine and glucocorticoid levels in a mouse model[J]. Oral Oncol. 2015;51 (11):991-997.

7. Thaker PH, Han LY, Kamat AA, et al. Chronic stress promotes tumor growth and angiogenesis in a mouse model of ovarian carcinoma[J]. Nat Med. 2006;12(8):939-944.

8. Strange KS, Kerr LR, Andrews HN, et al. Psychosocial stressors and mammary tumor growth: an animal model[J]. Neurotoxicol Teratol. 2000;22(1):89-102.
9. Gao G, Sun J, Gao J, et al. Chronic stress promoted the growth of ovarian carcinoma via increasing serum levels of norepinephrine and interleukin-10 and altering nm23 and NDRG1 expression in tumor tissues in nude mice[J]. Biosci Trends. 2013;7(1):56-63.

10. Willner P. Chronic mild stress (CMS) revisited: consistency and behavioural-neurobiological concordance in the effects of CMS[J]. Neuropsychobiology. 2005;52(2):90-110.

11. Suhail N, Bilal N, Hasan S, et al. Chronic unpredictable stress (CUS) enhances the carcinogenic potential of 7,12-dimethylbenz(a)anthracene (DMBA) and accelerates the onset of tumor development in Swiss albino mice[J]. Cell Stress Chaperones. 2015;20(6):1023-1036.

12. Suhail N, Bilal N, Hasan S, et al. Chronic unpredictable stress exacerbates 7,12-dimethylbenz (a) anthracene induced hepatotoxicity and nephrotoxicity in Swiss albino mice[J]. Mol Cell Biochem. 2011;355(1-2):117-126.

13. Katz RJ, Roth KA, Carroll BJ. Acute and chronic stress effects on open field activity in the rat: implications for a model of depression[J]. Neurosci Biobehav Rev. 1981;5(2):247-251.

14. Sitohy B, Nagy JA, Dvorak HF. Anti-VEGF/VEGFR therapy for cancer: reassessing the target[J]. Cancer Res. 2012;72(8):1909-1914.

15. Babjuk M, Bohle A, Burger $M$, et al. EAU Guidelines on Non-Muscle-invasive Urothelial Carcinoma of the Bladder: update 2016[J]. Eur Urol. 2017;71(3):447-461.

16. Barik J, Marti F, Morel C, et al. Chronic stress triggers social aversion via glucocorticoid receptor in dopaminoceptive neurons[J]. Science. 2013;339(6117):332-335.

17. Moshkov DA, Romanchenko SP, Parnyshkova EY, et al. Effect of dopamine on Ehrlich ascites carcinoma cells[J]. Bull Exp Biol Med. 2013;154(5):686-691.

18. Lutgendorf SK, Degeest K, C Y S, et al. Depression, social support, and beta-adrenergic transcription control in human ovarian cancer[J]. Brain Behav Immun. 2009;23(2):176-183.

19. Schrepf A, Thaker PH, Goodheart MJ, et al. Diurnal cortisol and survival in epithelial ovarian cancer[J]. Psychoneuroendocrinology. 2015;53:256-267.

20. Mcdonald PH, Lefkowitz RJ. Beta-Arrestins: new roles in regulating heptahelical receptors' functions[J]. Cell Signal. 2001;13 (10):683-689.

21. Bernabe DG, Tamae AC, Biasoli ER, et al. Stress hormones increase cell proliferation and regulates interleukin-6 secretion in human oral squamous cell carcinoma cells[J]. Brain Behav Immun. 2011;25(3):574-583.

22. Lutgendorf SK, Cole S, Costanzo E, et al. Stress-related mediators stimulate vascular endothelial growth factor secretion by two ovarian cancer cell lines[J]. Clin Cancer Res. 2003;9(12):4514-4521.

23. Zheng Y, Izumi K, Li Y, et al. Contrary regulation of bladder cancer cell proliferation and invasion by dexamethasone-mediated glucocorticoid receptor signals[J]. Mol Cancer Ther. 2012;11(12):2621-2632.

24. Wang J, Zhang X, Wei P, et al. Livin, Survivin and Caspase 3 as early recurrence markers in non-muscle-invasive bladder cancer[J]. World J Urol. 2014;32(6):1477-1484.

25. J A K, Lotan Y, Karakiewicz PI, et al. Use of combined apoptosis biomarkers for prediction of bladder cancer recurrence and mortality after radical cystectomy[J]. Lancet Oncol. 2007;8(2):128-136.

26. Wu TT, Chen JH, Lee YH, et al. The role of BCL-2, p53, and ki-67 index in predicting tumor recurrence for low grade superficial transitional cell bladder carcinoma[J]. $J$ Urol. 2000;163(3):758-760.

27. Margulis V, Lotan Y, Karakiewicz PI, et al. Multi-institutional validation of the predictive value of Ki-67 labeling index in patients with urinary bladder cancer[J]. J Natl Cancer Inst. 2009;101(2):114-119.

28. Wang L, Zhou M, Feng C, et al. Prognostic value of Ki67 and p63 expressions in bladder cancer patients who underwent radical cystectomy[J]. Int Urol Nephrol. 2016;48(4):495-501.

29. Nilsson MB, Armaiz-Pena G, Takahashi R, et al. Stress hormones regulate interleukin-6 expression by human ovarian carcinoma cells through a Src-dependent mechanism[J]. J Biol Chem. 2007;282 (41):29919-29926. 
30. Lutgendorf SK, Johnsen EL, Cooper B, et al. Vascular endothelial growth factor and social support in patients with ovarian carcinoma[J]. Cancer. 2002;95(4):808-815.

31. Costanzo ES, Lutgendorf SK, Sood AK, et al. Psychosocial factors and interleukin-6 among women with advanced ovarian cancer[J] Cancer. 2005;104(2):305-313.
32. Zhang $\mathrm{X}$, Zhang $\mathrm{Y}$, He Z, et al. Chronic stress promotes gastric cancer progression and metastasis: an essential role for ADRB2[J]. Cell Death Dis. 2019;10(11):788.

\section{Publish your work in this journal}

Psychology Research and Behavior Management is an international, peer-reviewed, open access journal focusing on the science of psychology and its application in behavior management to develop improved outcomes in the clinical, educational, sports and business arenas. Specific topics covered in the journal include: Neuroscience, memory and decision making; Behavior modification and management; Clinical applications; Business and sports performance management; Social and developmental studies; Animal studies. The manuscript management system is completely online and includes a very quick and fair peer-review system, which is all easy to use. Visit http://www. dovepress.com/testimonials.php to read real quotes from published authors. 